\title{
And If Bell's Inequality Were Not Violated
}

\section{Olivier Serret}

ESIM Engineer-60 rue de la Marne, Cugnaux, France

Email: o.serret@free.fr

Received 28 May 2014; revised 24 June 2014; accepted 18 July 2014

Copyright (C) 2014 by author and Scientific Research Publishing Inc.

This work is licensed under the Creative Commons Attribution International License (CC BY).

http://creativecommons.org/licenses/by/4.0/

c) (i) Open Access

\begin{abstract}
It briefly recalls the theory of Bell's inequality and some experimental measures. Then measurements are processed on one hand according to a property of the wave function, on the other hand according to the sum definition. The results of such processed measures are apparently not the same, so Bell's inequality would not be violated. It is a use of the wave function which implies the violation of the inequality, as it can be seen on the last flowcharts.
\end{abstract}

\section{Keywords}

Bell's Theorem, Bell's Inequality Violation, Entangled Photons, Quantum Sum, Experimental Measures, Wave Function

\section{Introduction}

Some quantum phenomena can cause astonishment. The violation of Bell's inequality is a part of the surprises raised by Quantum Mechanics [1] [2]. This violation means that it would be necessary to give up at least one of the following three assumptions: the principle of locality (two photons can not influence each other at a distance greater than the speed of light), the assumption of causality (to each effect at least one cause) and the assumption of realism (any particle has its own property). After recalling what Bell's inequality and experimental measures are, we will discuss how this inequality can be violated or not.

This paper is a criticism of Pr Alain Aspect's demonstration; it is neither a criticism of Bell's inequality theory, nor of its consequences here before.

\section{Experimental Measures of Bell's Inequality}

\subsection{The Experimental Set-Up}

Hereinafter proposed by Professor John Stewart BELL [3] the experimental set-up:

The source is a stream of 2 entangled photons: 
-the two photons leave in two opposite directions.

- "entangled" means that one photon is polarized along $\theta$ and the other photon along $(\theta+\pi)$, it means the entangled photons have the same polarity $( \pm \pi)$.

$A$ and $B$ are separators with switching function depending on the photon polarity (that is to say in the direction of the electric field associated with the photon).

$D A, D A^{\perp} D B$ and $D B^{\perp}$ are four independent photon detectors, or counters.

Experiments done in Paris, Innsbruck and Genève [4] with two-channel polarizers look like Figure 1.

\subsection{Measures of Entangled Photons}

-if the polarity of the photon is rather parallel to $\alpha$, the photon is leaving to $D A$ detector,

-otherwise, if the polarity of the photon is rather perpendicular to $\alpha$, the photon is leaving to $D A^{\perp}$ detector

-if the polarity of the other photon is rather parallel to $\beta$, the photon is leaving to $D B$

-otherwise, if the polarity of the other photon is rather perpendicular to $\beta$, the photon is leaving to $D B^{\perp}$

When a photon is detected the measurement is conventionally +1 , and when it is not detected the measurement is conventionally -1 .

Given:

$a_{i}=D A$ measurement (at the $\mathrm{i}^{\text {th }}$ throw)

$\bar{a}_{i}=D A^{\perp}$ measurement (at the $\mathrm{i}^{\text {th }}$ throw); $\bar{a}_{i}$ could also be written $a_{i}^{\perp}$

$b_{i}=D B$ measurement (at the $\mathrm{i}^{\text {th }}$ throw)

$\bar{b}_{i}=D B^{\perp}$ measurement (at the $i^{\text {th }}$ throw); $\bar{b}_{i}$ could also be written $b_{i}^{\perp}$

$\bar{a}_{i}$ is the complement of $a_{i}$ [5]: when the photon is detected by $D A$, it is not detected by $D A^{\perp}$ (and conversely)

$\bar{b}_{i}$ is the complement of $b_{i}$ : when the photon is detected by $D B$, it is not detected by $D B^{\perp}$ (and conversely)

At each entangled photon, by construction:

$$
a_{i}=-\bar{a}_{i}
$$

which means $1=-(-1)$ or $-1=-(1)$, either the photon is detected by $D A$, or by $D A^{\perp}$ and

$$
b_{i}=-\bar{b}_{i}
$$

which means $1=-(-1)$ or $-1=-(1)$, either the other photon is detected by $D B$, or by $D B^{\perp}$.

\subsection{Experimental Measures}

The measures are of the form:

1) If the two polarizers are oriented in the same direction, the two photons always behave the same way (transmitted or absorbed depending on the angle of the polarizer with the polarization).

2) If the two polarisers are inclined at an angle of $30^{\circ}$ with respect to each other, then the two photons have exactly the same behavior in $3 / 4$ cases and in opposite fourth cases.

3) If the two polarisers are inclined at an angle of $60^{\circ}$ with respect to each other, then the two photons have the same behavior in exactly $1 / 4$ cases and in opposed $3 / 4$ cases [6].

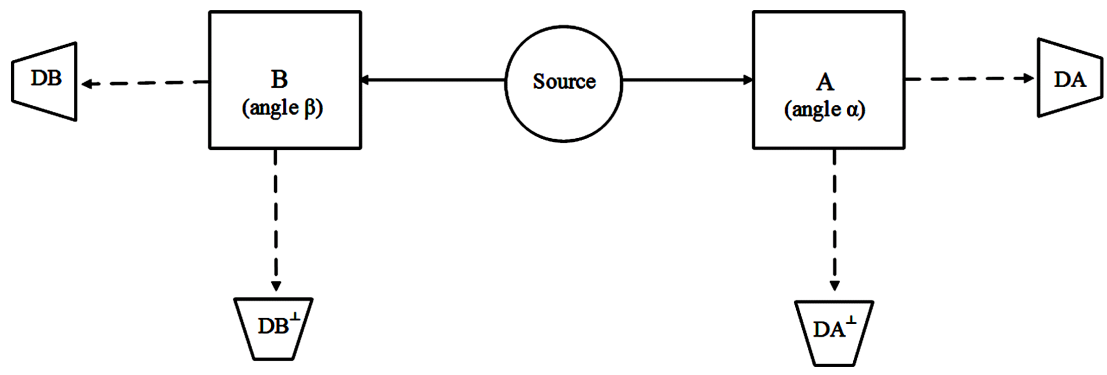

Figure 1. Bell's inequality set-up. 


\subsection{The Quantum Sum $s_{i}$}

Let us define the first quantum sum $s_{i}$ : "each pair of particles carries with it sufficient information to calculate the following number" [7]

$$
s_{i}=\left(a_{i}+\bar{a}_{i}\right) \cdot b_{i}+\left(a_{i}-\bar{a}_{i}\right) \cdot \bar{b}_{i}
$$

Previous quantum sum can also be written as:

$$
s_{i}=a_{i} \cdot b_{i}+\bar{a}_{i} \cdot b_{i}+a_{i} \cdot \overline{b_{i}}-\bar{a}_{i} \cdot \overline{b_{i}}
$$

Remark: another way to write the quantum sum is:

$$
s_{i}^{\prime}=a_{i} \cdot b_{i}^{\prime}-\bar{a}_{i} \cdot b_{i}^{\prime}+a_{i} \cdot \overline{b_{i}^{\prime}}+\bar{a}_{i} \cdot \overline{b_{i}^{\prime}}
$$

where $b_{i}$ has thus been noted $\overline{b_{i}^{\prime}}$ (and consequently $\overline{b_{i}}$ has been noted $b_{i}^{\prime}$ )

It is only a convention to call a detector $D B$ or $D B^{\perp}$, and so the measures $b_{i}$ or $\overline{b_{i}^{\prime}}$. In practice, it will change the sign of the result $s_{i}^{\prime}$, not its absolute value:

$$
\left|s_{i}\right|=\left|s_{i}^{\prime}\right|
$$

\subsection{Bell's Inequality Definition}

Let us remind what Bell's inequality definition is:

"Bell's theorem [or Bell's inequality] is not defined according to a clear statement that would be found in a reference article" [8]; so we will hereafter take the definitions given by Professor SCARANI:

"This is the statement of the Bell theorem: if our hypothesis is correct, the average value of $s_{i}$ must be between -2 and +2 . That's all..." [7].

Then Bell's therorem (or inequality) is mathematically transcribed to:

$$
-2 \leq S \leq+2
$$

with $S$ the average value of $s_{i}$

\subsection{Average Quantum Sum $S_{1}$}

The sum used in the updated Bell's inequality definition is: "making measurements on a large number of pairs, it can measure the average value of $s_{i}$ " [7].

Let us call "the average value of $s_{i}$ ": $S_{1}$

$$
S_{1}=A v \cdot\left[s_{i}\right]
$$

$S_{1}$ the average value of $s_{i}$ is "the algebraic sum of the four average values corresponding to the measures" [7], which is mathematically transcripted (see Appendix) by:

$$
S_{1}=A v \cdot\left[a_{i} \cdot b_{i}\right]+A v \cdot\left[\bar{a}_{i} \cdot b_{i}\right]+A v \cdot\left[a_{i} \cdot \bar{b}_{i}\right]-A v \cdot\left[\bar{a}_{i} \cdot \bar{b}_{i}\right]
$$

\section{Treatment of the Measures}

\subsection{Treatment from a Wave Function Property}

Pr. Valerio Scarani [9] [10], popularizing Pr Aspect's demonstration, proceeds starting from the wave function and according to quantum calculation ending up to a property [7]:

$$
S_{2}(\alpha, \beta)=E(\alpha, \beta)+E(\alpha+\pi / 2, \beta)+E(\alpha, \beta+\pi / 2)-E(\alpha+\pi / 2, \beta+\pi / 2)
$$

with

$$
E(\alpha, \beta)=-\cos (\alpha, \beta)
$$

\section{Important remark:}

This sum $S_{2}$ looks like the average sum $S_{1}$ but it is not the same:

$\diamond S_{1}$ is function of experimental measures $\left(a_{i}, b_{i}\right)$ [cf. Equation (9)]; $\left(a_{i}, b_{i}\right)$ are a couple of numbers: 


$$
\left(a_{i}, b_{i}\right)=( \pm 1, \pm 1)
$$

$\diamond S_{2}$ is function of experimental conditions $(\alpha, \beta)$ [cf. Equation (10)]; $(\alpha, \beta)$ are a couple of angles:

$$
(\alpha, \beta) \in[0 ; 2 \pi]^{2}
$$

Starting again with Equation (10) and Equation (11),

$$
\begin{gathered}
S_{2}(\alpha, \beta)=-\cos (\alpha+\beta)-\cos (\alpha+\pi / 2+\beta)-\cos (\alpha+\beta+\pi / 2)+\cos (\alpha+\pi / 2+\beta+\pi / 2) \\
S_{2}(\alpha, \beta)=-\cos (\alpha+\beta)-\sin (\alpha+\beta)-\sin (\alpha+\beta)-\cos (\alpha+\beta) \\
S_{2}(\alpha, \beta)=-2[\cos (\alpha+\beta)+\sin (\alpha+\beta)]
\end{gathered}
$$

For example, for $\alpha=0^{\circ}$ and $\beta=30^{\circ}$ :

$$
\begin{aligned}
& S_{2}(\alpha, \beta)=-2[0.87+0.5]=-2.7 \\
& S_{2}\left(\alpha=0^{\circ}, \beta=30^{\circ}\right) \leq-2
\end{aligned}
$$

It would be in this case $\left(\alpha=0^{\circ}, \beta=30^{\circ}\right)$, according to Equation (10) and Equation (11), violation of Bell's inequality.

Remark: the concern is that this result is the consequence of theoretical calculations dealing with angles $\alpha$ and $\beta$ of the test conditions; it is not the result of measures $a_{i}$ and $b_{i}$ experimentally found (cf Table A2 in Appendix).

\subsection{Quantum Sum from the Experimental Measures in a Particular Case}

In the previous case where $\left(\alpha=0^{\circ}, \beta=30^{\circ}\right)$, returning to the experimental measures [cf Table A2 in Appendix], we get Table 1 .

According to the sum definition [cf. Equation (8)],

$$
\text { for }\left(\alpha=0^{\circ}, \beta=30^{\circ}\right), S_{1}=A v \cdot\left[s_{i}\right]=-8 / 8=-1
$$

or according to the sum property [cf. Equation (9)],

$$
\text { for }\left(\alpha=0^{\circ}, \beta=30^{\circ}\right), S_{1}=(+4 / 8)+(-4 / 8)+(-4 / 8)-(+4 / 8)=-8 / 8=-1
$$

id est:

$$
-2 \leq S_{1}\left(\alpha=0^{\circ}, \beta=30^{\circ}\right) \leq+2
$$

For $\left(\alpha=0^{\circ}, \beta=30^{\circ}\right)$, the average sum $S_{1}$ does not violate Bell's inequality [as defined in Equation (7)].

Table 1. Experimental measures with an angle of $30^{\circ}$ processed.

\begin{tabular}{crrrcccc}
\hline$\# \quad$ & Angle $\alpha$ & Angle $\beta$ & $a_{i} \cdot b_{i}$ & $\bar{a}_{i} \cdot b_{i}$ & $a_{i} \cdot \bar{b}_{i}$ & $\bar{a}_{i} \cdot \bar{b}_{i}$ & $s_{i}$ \\
\hline 3 & 0 & $30^{\circ}$ & 1 & -1 & -1 & 1 & -2 \\
4 & 0 & $30^{\circ}$ & 1 & -1 & -1 & 1 & -2 \\
5 & 0 & $30^{\circ}$ & 1 & -1 & -1 & 1 & -2 \\
6 & 0 & $30^{\circ}$ & -1 & 1 & 1 & -1 & 2 \\
7 & 0 & $30^{\circ}$ & 1 & -1 & -1 & 1 & -2 \\
8 & 0 & $30^{\circ}$ & 1 & -1 & -1 & 1 & -2 \\
9 & 0 & $30^{\circ}$ & 1 & -1 & -1 & 1 & -2 \\
10 & 0 & $30^{\circ}$ & -1 & 1 & 1 & -1 & 2 \\
Average & & & $+\mathbf{4 / 8}$ & $-\mathbf{4 / 8}$ & $-\mathbf{4 / 8}$ & $+\mathbf{4 / 8}$ & $-\mathbf{8 / 8}$ \\
\hline
\end{tabular}




\subsection{Quantum Sum from the Experimental Measures in the General Case}

Let us process the three tables (Tables A1-A3) from Appendix into Table 2.

We can verify that for all experimental measures cf. Equation (9):

$$
\begin{array}{r}
S_{1}=A v \cdot\left[a_{i} \cdot b_{i}\right]+A v \cdot\left[\bar{a}_{i} \cdot b_{i}\right]+A v \cdot\left[a_{i} \cdot \bar{b}_{i}\right]-A v \cdot\left[\bar{a}_{i} \cdot \bar{b}_{i}\right] \\
S_{1}=(+2 / 18)+(-2 / 18)+(-2 / 18)-(+2 / 18)=-4 / 18=-0.22
\end{array}
$$

If we processed from the definition for all experimental measures $\mathrm{cf}$. Equation (8):

$$
\begin{aligned}
S_{1}=A v \cdot\left[s_{i}\right] \\
\quad S_{1}=-4 / 18=-0.22
\end{aligned}
$$

so processing all experimental measures

$$
-2 \leq S_{1} \leq+2
$$

With all the measures, the average sum $S_{1}$ does not violate Bell's inequality [as defined in Equation (7)]. So, in the particular case or in general, using the definition $S_{1}$, Bell's inequality is never violated.

\begin{tabular}{|c|c|c|c|c|c|c|c|}
\hline$\# i$ & Angle $\alpha$ & Angle $\beta$ & $a_{i} \cdot b_{i}$ & $\bar{a}_{i} \cdot b_{i}$ & $a_{i} \cdot \bar{b}_{i}$ & $\bar{a}_{i} \cdot \bar{b}_{i}$ & $s_{i}$ \\
\hline 1 & 0 & $0^{\circ}$ & 1 & -1 & -1 & 1 & -2 \\
\hline 2 & 0 & $0^{\circ}$ & 1 & -1 & -1 & 1 & -2 \\
\hline 3 & 0 & $30^{\circ}$ & 1 & -1 & -1 & 1 & -2 \\
\hline 4 & 0 & $30^{\circ}$ & 1 & -1 & -1 & 1 & -2 \\
\hline 5 & 0 & $30^{\circ}$ & 1 & -1 & -1 & 1 & -2 \\
\hline 6 & 0 & $30^{\circ}$ & -1 & 1 & 1 & -1 & 2 \\
\hline 7 & 0 & $30^{\circ}$ & 1 & -1 & -1 & 1 & -2 \\
\hline 8 & 0 & $30^{\circ}$ & 1 & -1 & -1 & 1 & -2 \\
\hline 9 & 0 & $30^{\circ}$ & 1 & -1 & -1 & 1 & -2 \\
\hline 10 & 0 & $30^{\circ}$ & -1 & 1 & 1 & -1 & 2 \\
\hline 11 & 0 & $30^{\circ}$ & -1 & 1 & 1 & -1 & 2 \\
\hline 12 & 0 & $60^{\circ}$ & -1 & 1 & 1 & -1 & 2 \\
\hline 13 & 0 & $60^{\circ}$ & -1 & 1 & 1 & -1 & 2 \\
\hline 14 & 0 & $60^{\circ}$ & 1 & -1 & -1 & 1 & -2 \\
\hline 15 & 0 & $60^{\circ}$ & -1 & 1 & 1 & -1 & 2 \\
\hline 16 & 0 & $60^{\circ}$ & -1 & 1 & 1 & -1 & 2 \\
\hline 17 & 0 & $60^{\circ}$ & -1 & 1 & 1 & -1 & 2 \\
\hline 18 & 0 & $60^{\circ}$ & 1 & -1 & -1 & 1 & -2 \\
\hline Average & & & $+2 / 18$ & $-2 / 18$ & $-2 / 18$ & $+2 / 18$ & $-4 / 18$ \\
\hline
\end{tabular}

\subsection{Synthesis about the Two Arguments}

Paragraphs 3.1 and 3.2 can be boiled out to the flowchart of Figure 2.

And from a more general point of view, the synthesis flowchart will be Figure 3.

Table 2. Experimental measures processed. 


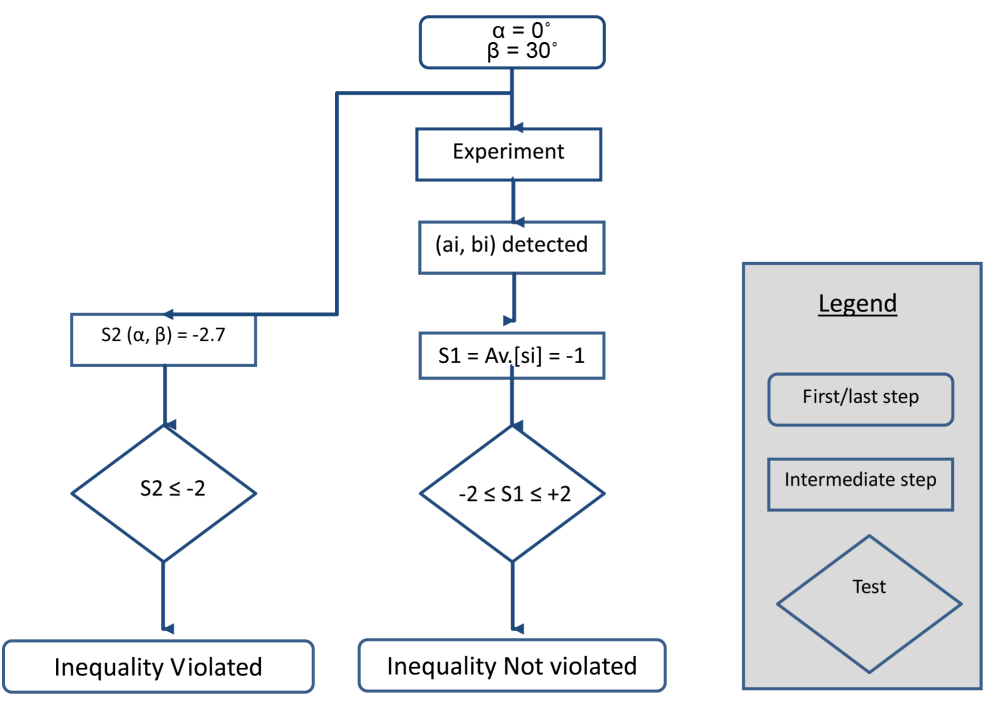

Figure 2. Flowchart of the 2 arguments.

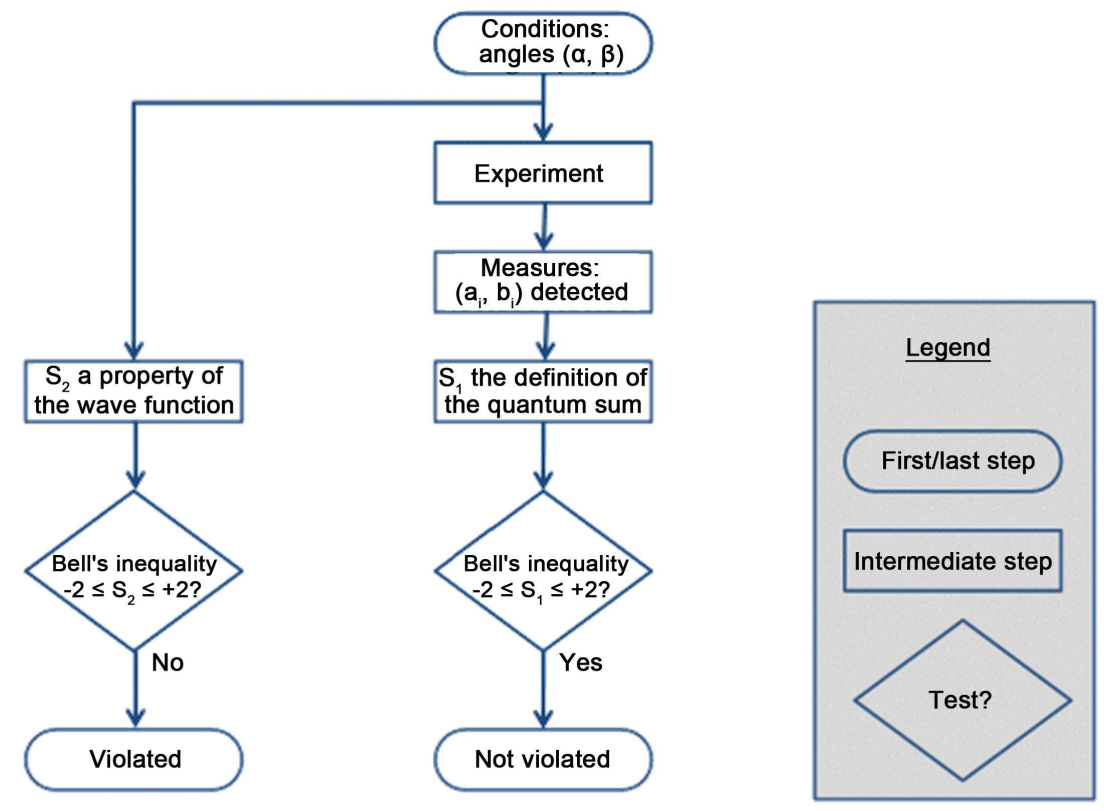

Figure 3. Synthetic flowchart.

\subsection{Application to the Aspect's Experiment of 1983}

In present paper, analysis has been done mainly from Dr. Scarani presentation, according to the property

$$
S_{2}(\alpha, \beta)=E(\alpha, \beta)+E(\alpha+\pi / 2, \beta)+E(\alpha, \beta+\pi / 2)-E(\alpha+\pi / 2, \beta+\pi / 2)
$$

[cf Equation10] with $E(\alpha, \beta)=-\cos (\alpha+\beta)$ [cf. Equation (11)]

Then the extremum are for values as $\pi / 6$ or $30^{\circ}$ [cf. Equation (18)], and $\pi / 3$ or $60^{\circ}$.

It is interesting to note than in Aspect's experiment of 1983 [3], the extremum were for values as $\pi / 8$ (or $22.5^{\circ}$ ) and $3 \pi / 8$ (or $67.5^{\circ}$ ). The reason comes the experiment was different (in BCHSH experiment the difference between two angles are not $\pi / 2$ but are $\pi / 4$ ), and so property was different:

$$
S_{3}(\theta)=3 \cos (2 \theta)-\cos (6 \theta)
$$

with 


$$
\theta=(a, b)
$$

Let us correlate 1983 notation with present notation (see Table 3):

$$
s_{i}=A(\lambda, a) \cdot B(\lambda, b)-A(\lambda, a) \cdot B\left(\lambda, b^{\prime}\right)+A\left(\lambda, a^{\prime}\right) \cdot B(\lambda, b)+A\left(\lambda, a^{\prime}\right) \cdot B\left(\lambda, b^{\prime}\right)
$$

which is equivalent to

$$
s_{i}=\bar{a}_{i} \cdot b_{i}-\bar{a}_{i} \cdot \bar{b}_{i}+a_{i} \cdot b_{i}+a_{i} \cdot \bar{b}_{i}
$$

Equation (28) is exactly the same than the Equation (4) for the definition of Bell's inequality.

So we can use the same flowchart (Figure 4) to explain the difference of results between the definition of Bell's inequality and the property of a wave function: Starting from the experiment and the definition of $S_{1}$, Bell's inequality is not violated.

\section{Conclusion}

After recalling what the Bell's inequality and the experimental measures are, the violation of this inequality appears to have been obtained by processing from a wave function property and the experimental conditions, but

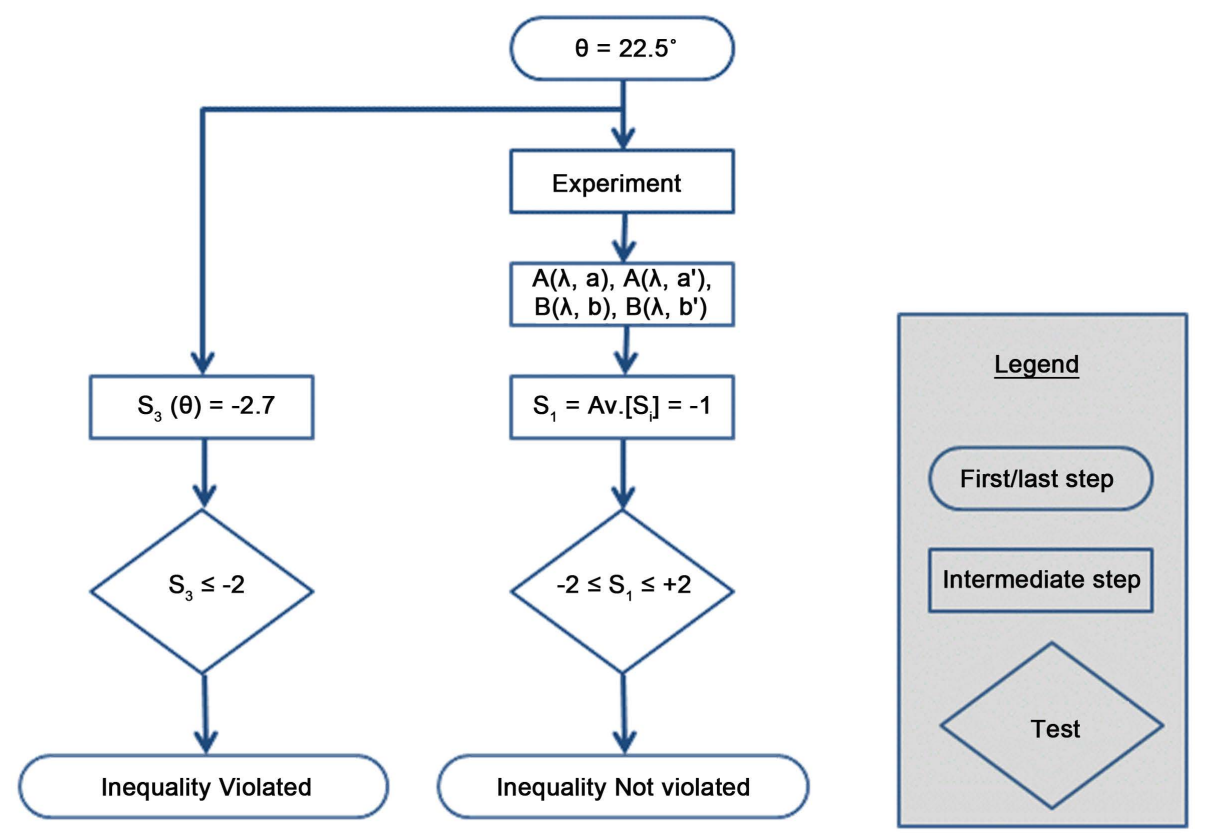

Figure 4. 1983 demonstration flowchart.

Table 3. Notation comparison.

\begin{tabular}{ccc} 
& 1983 notation & Present notation \\
\hline Separator angles & $a, b$ & $\alpha, \beta$ \\
Angle $\theta=(a, b)$ & $\theta=b-a$ & $\theta=\beta-\alpha$ \\
Property & $S_{3}(\theta)=3 \cos (2 \theta)-\cos (6 \theta)$ & $S_{3}(\theta)=3 \cos (2 \theta)-\cos (6 \theta)$ \\
Polarisation measure from A & $A(\lambda, a)$ & $\bar{a}_{i}$ \\
Polarisation measure from A & $A\left(\lambda, a^{\prime}\right)$ & $a_{i}$ \\
Polarisation measure from B & $B(\lambda, b)$ & $b_{i}$ \\
Polarisation measure from B & $B\left(\lambda, b^{\prime}\right)$ & $\bar{b}_{i}$ \\
Definition & See Equation (27) & See Equation (28) \\
\hline
\end{tabular}


have obscured the measures themselves. By treating these measures from the definition of $S$ (average sum of $s_{i}$ ), Bell's inequality appears to be respected. The ways of the two arguments are summarized in flowcharts.

This article is limited to the demonstration of the violation of Bell's inequality. It is neither a criticism of Bell's inequality theory, nor of its consequences.

\section{Acknowledgments}

I would like to thank Pr. Roger BOUDET for his encouragements and help in the diffusion of this paper, Pr. Claude DAVIAU for his critical reading, and the referees for their valuable suggestions.

\section{References}

[1] Greenberger, D.M., Horne, M.A. and Zeilinger, A. (2007) arXiv: 0712.0921. http://arxiv.org/abs/0712.0921

[2] Bell, J.S. (1964) Physics, 1, 195.

[3] Bell, J.S. (1966) Reviews of Modern Physics, 38, 447.

[4] Rowe, M.A., Kielpinski, D., Meyer, V., Sackett, C.A., Itano, W.M., Monroe, C. and Wineland, D.J. (2001) Nature, 409, 791. http://galileo.phys.virginia.edu/research/groups/sackett/publications/rowe 01.pdf

[5] Serres, M. and Farouki, N. (1997) Le Trésor. Flammarion, 640-642.

[6] Inégalité de Bell, Wikipedia. http://fr.wikipedia.org/wiki/In\%C3\%A9galit\%C3\%A9s de Bell\#Pr.C3.A9sentation et formulation simplifi.C3.A9e des_in.C3.A9galit.C3.A9s_de_Bell

[7] (2006) Valerio Scarani: “Initiation à la Physique Quantique”, Vuibert, 79, 80 and 119.

[8] (1983) Alain Aspect: “Thèse de doctorat” 27 to 36. http://tel.archives-ouvertes.fr/tel-00011844/

[9] Scarani, V., Chua, L. and Liu, S.Y. (2010) Six Quantum Pieces. World Scientific.

[10] Fitzsimons, J.F., Rieffel, E.G. and Scarani, V. (2012) arXiv: 1206.0785. http://arxiv.org/abs/1206.0785

[11] Bell's Theorem, Wikipedia. http://en.wikipedia.org/wiki/Bell's theorem 


\section{Appendix}

\section{A1. Bell's Inequality}

Let us remind the evolution of Bell's inequality.

The inequality that Bell derived can be written as:

$$
\rho(a, c)-\rho(a, b)-\rho(b, c) \leq 1
$$

where $\rho$ is the correlation between measurements of the spins of the pair of particles and $a, b$ and $c$ refer to three arbitrary settings of the two analysers [11].

Generalizing Bell's original inequality, it has been introduced the CHSH inequality, without any assumption of perfect correlations (or anti-correlations) at equal settings

$$
\rho(a, b)+\rho\left(a, b^{\prime}\right)+\rho\left(a^{\prime}, b\right)-\rho\left(a^{\prime}, b^{\prime}\right) \leq 2
$$

where $\rho$ denotes correlation in the quantum physicist's sense: "the expected value of the product of the two binary (+/-1 valued) outcomes... the CHSH inequality reduces to the original Bell inequality" [11].

\section{A2. Average Quantum Sum $S_{1}$}

Let us call "the average value of $s_{i}$ ”: $S_{1}$

$$
\begin{gathered}
S_{1}=A v \cdot\left[s_{i}\right] \\
S_{1}=\frac{1}{n} \sum_{i=1}^{i=n} s_{i}
\end{gathered}
$$

$S_{1}$ the average value of $s_{i}$ is "the algebraic sum of the four average values corresponding to the measures" [7], which is mathematically transcripted by:

$$
S_{1}=A v \cdot\left[a_{i} \cdot b_{i}\right]+A v \cdot\left[\bar{a}_{i} \cdot b_{i}\right]+A v \cdot\left[a_{i} \cdot \bar{b}_{i}\right]-A v \cdot\left[\bar{a}_{i} \cdot \bar{b}_{i}\right]
$$

Demonstration:

$$
S_{1}=\frac{1}{n} \sum_{i=1}^{i=n} s_{i}
$$

so

$$
\begin{gathered}
S_{1}=\frac{1}{n} \sum_{i=1}^{i=n}\left[\left(a_{i}+\bar{a}_{i}\right) \cdot b_{i}\right]+\left[\left(a_{i}-\bar{a}_{i}\right) \cdot \bar{b}_{i}\right] \\
S_{1}=\frac{1}{n} \sum_{i=1}^{i=n}\left(a_{i} \cdot b_{i}\right)+\frac{1}{n} \sum_{i=1}^{i=n}\left(\bar{a}_{i} \cdot b_{i}\right)+\frac{1}{n} \sum_{i=1}^{i=n}\left(a_{i} \cdot \bar{b}_{i}\right)+\frac{1}{n} \sum_{i=1}^{i=n}\left(-\bar{a}_{i} \cdot \bar{b}_{i}\right)
\end{gathered}
$$

and so

$$
S_{1}=A v \cdot\left[a_{i} \cdot b_{i}\right]+A v \cdot\left[\bar{a}_{i} \cdot b_{i}\right]+A v \cdot\left[a_{i} \cdot \bar{b}_{i}\right]-A v \cdot\left[\bar{a}_{i} \cdot \bar{b}_{i}\right]
$$

So $S_{1}$, average value of $s_{i}$, is effectively the algebraic [quantum] sum of the four average values. It is an equivalent definition.

\section{A3. Experimental Measures}

Let us translate these synthetic measures through examples.

a) If the two polarizers are oriented in the same direction, the two photons always behave the same way (transmitted or absorbed depending on the angle of the polarizer with the polarization): $\left\{a_{i}=b_{i}\right\}$ in all cases (Table A1).

b) If the two polarisers are inclined at an angle of $30^{\circ}$ with respect to each other, then the two photons have exactly the same behavior in 3/4 cases and in opposite fourth cases: $\left\{a_{i}=b_{i}\right\}$ in 3/4 cases (Table A2).

c) If the two polarisers are inclined at an angle of $60^{\circ}$ with respect to each other, then the two photons have the same behavior in exactly $1 / 4$ cases and in opposed 3/4 cases: $\left\{a_{i}=b_{i}\right\}$ in $1 / 4$ cases (Table A3). 
Table A1. Experimental measures with an angle of $0^{\circ}$.

\begin{tabular}{rrrrrrr}
\hline$\# i$ & Angle $\alpha$ & Angle $\beta$ & $a_{i}$ & $\bar{a}_{i}$ & $b_{i}$ & $\bar{b}_{i}$ \\
\hline 1 & 0 & $0^{\circ}$ & 1 & -1 & 1 & -1 \\
2 & 0 & $0^{\circ}$ & -1 & 1 & -1 & 1 \\
\hline
\end{tabular}

Table A2. Experimental measures with an angle of $30^{\circ}$.

\begin{tabular}{rrrrrrr}
\hline$\# i$ & Angle $\alpha$ & Angle $\beta$ & $a_{i}$ & $\bar{a}_{i}$ & $b_{i}$ & $\bar{b}_{i}$ \\
\hline 3 & 0 & $30^{\circ}$ & 1 & -1 & 1 & -1 \\
4 & 0 & $30^{\circ}$ & 1 & -1 & 1 & -1 \\
5 & 0 & $30^{\circ}$ & 1 & -1 & 1 & -1 \\
6 & 0 & $30^{\circ}$ & 1 & -1 & $-\mathbf{1}$ & $\mathbf{1}$ \\
7 & 0 & $30^{\circ}$ & -1 & 1 & -1 & 1 \\
8 & 0 & $30^{\circ}$ & -1 & 1 & -1 & 1 \\
9 & 0 & $30^{\circ}$ & -1 & 1 & -1 & 1 \\
10 & 0 & $30^{\circ}$ & -1 & 1 & $\mathbf{1}$ & $-\mathbf{1}$
\end{tabular}

Table A3. Experimental measures with an angle of $60^{\circ}$.

\begin{tabular}{rrrrrrr}
$\# i$ & Angle $\alpha$ & Angle $\beta$ & $a_{i}$ & $\bar{a}_{i}$ & $b_{i}$ & $\bar{b}_{i}$ \\
\hline 11 & 0 & $60^{\circ}$ & 1 & -1 & $-\mathbf{1}$ & $\mathbf{1}$ \\
12 & 0 & $60^{\circ}$ & 1 & -1 & $-\mathbf{1}$ & $\mathbf{1}$ \\
13 & 0 & $60^{\circ}$ & 1 & -1 & $-\mathbf{1}$ & $\mathbf{1}$ \\
14 & 0 & $60^{\circ}$ & 1 & -1 & 1 & -1 \\
15 & 0 & $60^{\circ}$ & -1 & 1 & $\mathbf{1}$ & $-\mathbf{1}$ \\
16 & 0 & $60^{\circ}$ & -1 & 1 & $\mathbf{1}$ & $-\mathbf{1}$ \\
17 & 0 & $60^{\circ}$ & -1 & 1 & $\mathbf{1}$ & $-\mathbf{1}$ \\
18 & 0 & $60^{\circ}$ & -1 & 1 & -1 & 1
\end{tabular}

More generally, the probability that the behavior of photons to be identical is

$$
\operatorname{Probability}\left(a_{i}=b_{i}\right)=\cos ^{2}(\beta-\alpha)
$$

with $(\beta-\alpha)$ the relative angle of the two polarizers.

a) When $\alpha=\beta=0^{\circ}$,

$$
\operatorname{Probability}\left(a_{i}=b_{i}\right)=\cos ^{2}\left(0^{\circ}-0^{\circ}\right)=1=100 \%
$$

b) When $\alpha=0^{\circ}$ and $\beta=30^{\circ}$,

$$
\text { Probability }\left(a_{i}=b_{i}\right)=\cos ^{2}\left(0^{\circ}-30^{\circ}\right)=(\sqrt{3} / 2)^{2}=3 / 4=75 \%
$$

c) When $\alpha=0^{\circ}$ and $\beta=60^{\circ}$,

$$
\operatorname{Probability}\left(a_{i}=b_{i}\right)=\cos ^{2}\left(0^{\circ}-60^{\circ}\right)=(1 / 2)^{2}=25 \%
$$


Scientific Research Publishing (SCIRP) is one of the largest Open Access journal publishers. It is currently publishing more than 200 open access, online, peer-reviewed journals covering a wide range of academic disciplines. SCIRP serves the worldwide academic communities and contributes to the progress and application of science with its publication.

Other selected journals from SCIRP are listed as below. Submit your manuscript to us via either submit@scirp.org or Online Submission Portal.
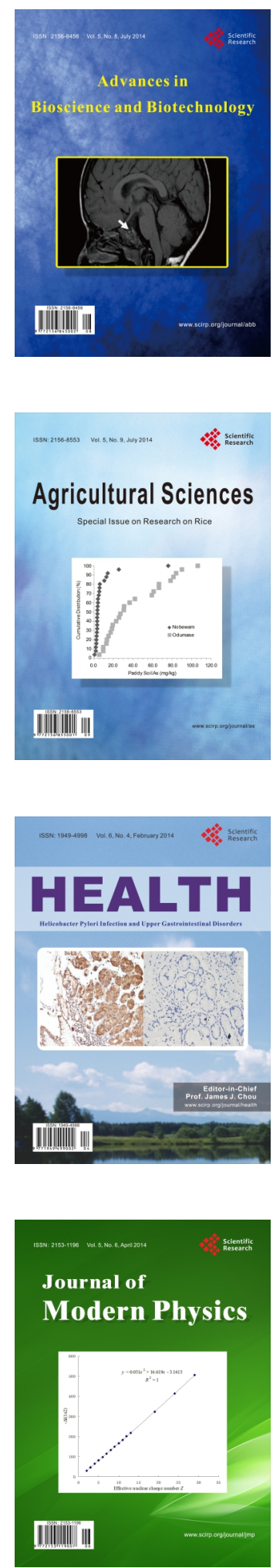
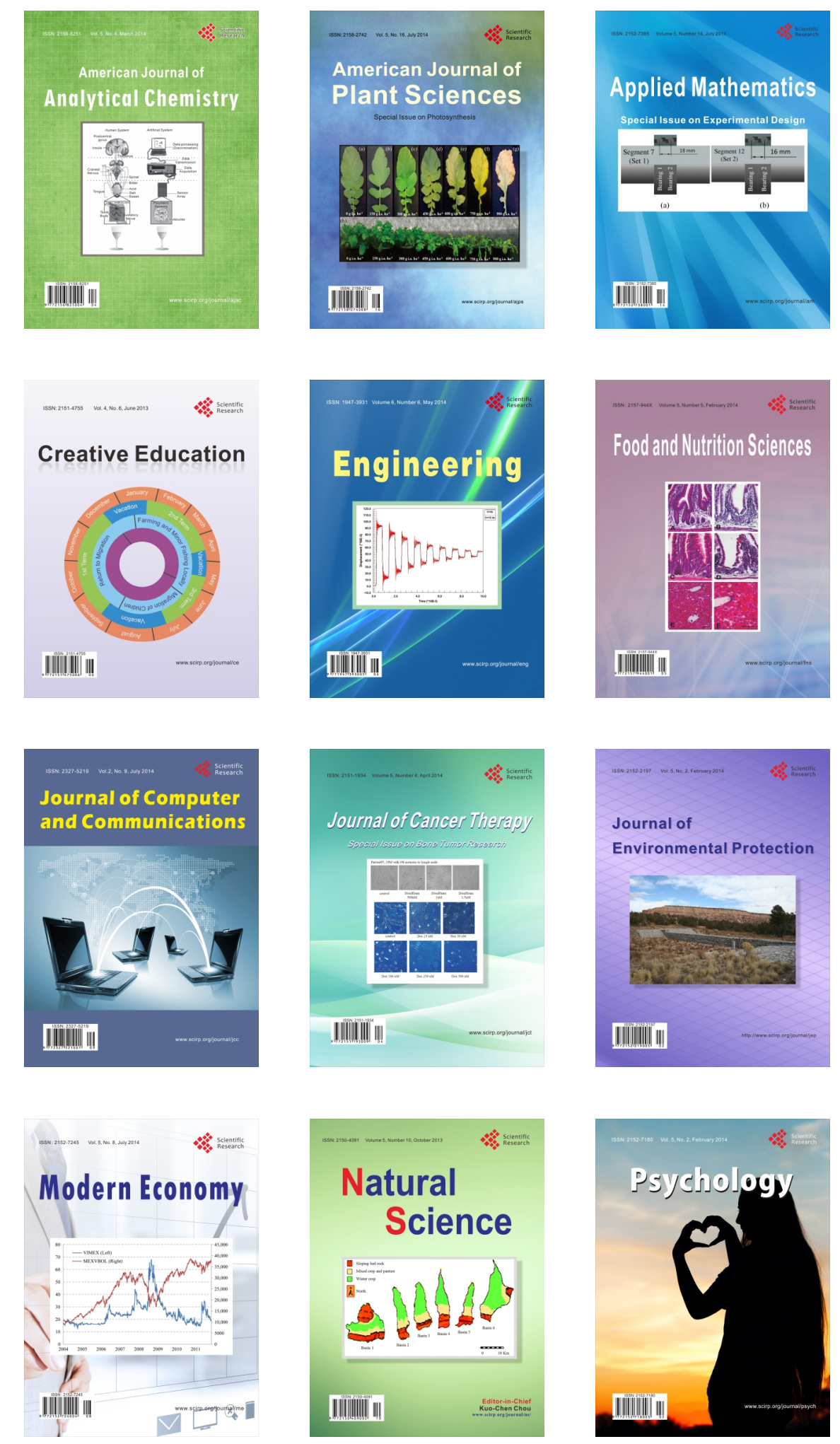